\title{
Efecto de la Adición de Biodiésel en el Rendimiento y la Opacidad de un Motor Diésel
}

\author{
Juan C. Rocha-Hoyos*, Edilberto A. Llanes-Cedeño, Santiago F. Celi-Ortega y Diana C. Peralta-Zurita \\ Univ. Internacional SEK, Fac. de Arquitectura e Ingenierías, Programa Desarrollo Tecnológico UISEK, Casilla 17-03-02, \\ Quito-Ecuador (e-mail: carlos.rocha@uisek.edu.ec; antonio.llanes@uisek.edu.ec)
}

* Autor a quien debe ser dirigida la correspondencia

Recibido Oct. 9, 2018; Aceptado Dic. 17, 2018; Versión final Ene. 16, 2019, Publicado Jun. 2019

\section{Resumen}

El objetivo principal de este trabajo es evaluar las prestaciones mecánicas y de opacidad de los combustibles diésel y mezclas de diésel/biodiésel en vehículos tipo camioneta a $2810 \mathrm{msnm}$, mediante pruebas dinamométricas. Para el estudio, se seleccionaron dos camionetas en las que se experimentaron cuatro mezclas: B10 (10\% biodiésel), B20 (20\% biodiésel), B10A (10\% biodiésel con aditivos), y B20A (20\% biodiésel con aditivos). Se determina la incidencia en el rendimiento mecánico del vehículo a partir de la realización de pruebas de potencia, par motor y opacidad. Se obtiene que la mezcla B10A es la más óptima para reemplazar al diésel puro, logrando mantener el par motor y la potencia sin variaciones significativas con relación al diésel para un nivel del $95.0 \%$ de confianza. Se concluye que a medida que aumenta el porcentaje de biodiésel se disminuye en $2.37 \%$ el par motor y en $1.37 \%$ la potencia con relación al diésel. Además, los combustibles con mezclas de biodiésel y con la adicción de aditivo reducen la emanación de hollín bajo el criterio de opacidad hasta aproximadamente un $37 \%$.

Palabras clave: biodiésel; motores diésel; aditivo de combustible; rendimiento del motor; hollín

\section{Effect of the Addition of Biodiesel on the Performance and Opacity of a Diesel Engine}

\begin{abstract}
The main objective of this work is to evaluate the mechanical and opacity performance of diesel fuels and diesel/biodiesel mixtures in van vehicles at 2810 meters above sea level, by means of dynamometric tests. For the study, two van in which four mixtures were evaluated: B10 (10\% biodiesel), B20 (20\% biodiesel), B10A (10\% biodiesel with additives), and B20A (20\% biodiesel with additives). The impact on the mechanical performance of the vehicle from testing of power, torque and opacity were determined. It is obtained that the B10A mixture is the optimum one to replace the pure diesel, managing to maintain the torque and power without significant variations in relation to the pure diesel for a level of $95.0 \%$ confidence. It was concluded that as the percentage of biodiesel increases, engine torque decreases by $2.37 \%$ and the power by $1.37 \%$ in relation to pure diesel. In addition, fuels with biodiesel mixtures and with additives reduce soot emission under the opacity criterion up to approximately $37 \%$.
\end{abstract}

Keywords: biodiesel; diesel engines; fuel additives; motor performance; soot 


\section{INTRODUCCIÓN}

La economía de cada país depende directa o indirectamente de los recursos petrolíferos a base de fósiles, reduciéndose gradualmente mediante la industrialización masiva, el transporte y la explosión demográfica (Afzal et al., 2018). Además, las preocupaciones por los desequilibrios climáticos, el calentamiento global del planeta y los compromisos adquiridos por los países desarrollados para mejorar la seguridad del suministro energético y fomentar el uso de las energías renovables son solo algunos de los factores que convierten al biodiésel en una interesante alternativa (Rocha-Hoyos et al., 2017). El biodiésel, el etanol, el metanol son los principales combustibles alternativos prospectivos que pueden reducir el calentamiento global, el consumo de combustibles fósiles y las emisiones de escape. Actualmente, se genera una masiva búsqueda del uso de aceite vegetal en los motores diésel seguido de la utilización del biodiésel con características técnicamente mejoradas en comparación con los aceites vegetales (Chen et al., 2018). Generalmente, la producción de biodiésel a través de la transesterificación de aceites vegetales es el método más reconocido y aceptado en todo el mundo (Mumtaz et al., 2012). Esté se sintetiza a partir de materia prima con un alto contenido de ácidos grasos insaturados, como los ácidos linoleico y linolénico, es más susceptible a la oxidación, considerando las modificaciones de algunas de sus propiedades como mayor número de cetano, punto de inflamabilidad y características de lubricación (Mofijur et al., 2014; Payri et al., 2011).

Las emisiones y el consumo de combustible se han reducido significativamente en virtud de los esfuerzos en diseño de motores (Mena et al., 2018). Sin embargo, es difícil alcanzar los estándares de emisión requeridos únicamente a través del diseño del motor. Se intentan métodos alternativos para obtener niveles de emisión más bajos, mejores prestaciones del motor y aumento de la durabilidad del combustible mediante la mezcla de diferentes aditivos dentro del combustible diésel (Moghaddam y Moghaddam, 2014). Durante el trabajo de investigación actual, diferentes extractos naturales y antioxidantes áticos se usan como aditivos, evaluando su impacto. Los antioxidantes sintéticos se usaron como aditivos (tocoferoles, butil hidroxil tolueno (BHT), tbutil hidroquinona (TBHQ), etc.) para mejorar la estabilidad oxidativa del biodiesel (Afzal et al., 2018). Los datos experimentales reportados por varios investigadores revelaron que la combustión del biodiésel resulta en niveles reducidos de partículas y emisiones de monóxido de carbono, pero niveles ligeramente más altos de emisiones de óxido de nitrógeno (NOx) en comparación con los resultantes de la combustión de diésel convencional (Thiyagarajan et al., 2017; Wei et al., 2018). Además, varios investigadores han estudiado el control de las emisiones de NOx y han informado de impactos significativos (Varatharajan et al., 2011), y el vínculo sustancial entre la composición del biodiésel y los niveles de emisión (Ozsezen et al., 2009; Qi et al., 2009; Thoo et al., 2014). Según Lenin et al. (2013) menciona que estos aditivos crean una acción catalítica para permitir una mejor combustión de hidrocarburos. Las características de rendimiento y emisión del combustible se analizan e indican que los aditivos reducen el retardo de encendido y el consumo específico de combustible al tiempo que aumentan los valores térmicos del combustible (Basha y Gopal, 2012). Se informó que la reducción de las emisiones de $\mathrm{CO}$ y humo estaba altamente relacionada con el contenido de oxígeno del biodiésel y el efecto catalizador de los aditivos de combustible a base de metal (Çelik et al., 2015; Rocha-Hoyos et al., 2018).

La investigación inicia frente a los antecedentes manifestados, por los altos niveles de contaminación y polución generados especialmente por el parque automotriz, frente al escaso aprovechamiento energético de las materias orgánicas como fuentes de energía y la producción de biocombustibles que garanticen un óptimo funcionamiento en motores de combustión interna. Al poder realizar una producción de biodiésel y utilizarlo como aditivo para los vehículos que utilizan como combustible el diésel fósil. El objetivo principal de este trabajo es evaluar las prestaciones mecánicas y de opacidad de los combustible diésel y mezclas de diésel/biodiésel en vehículos tipo camioneta a $2810 \mathrm{msnm}$, mediante pruebas dinamométricas para la propuesta de una alternativa en el uso de estos combustibles

\section{METODOLOGÍA}

El trabajo experimental se basa en dos etapas: la primera es la determinación de las principales propiedades del combustible, así como de las mezclas de estudio; y la segunda etapa corresponde al estudio del comportamiento mecánico y opacidad del motor diésel operado con diésel y mezclas diésel/biodiésel (B10, B20), además de diésel/biodiésel con aditivo (B10A, B20A). Este procedimiento se realiza a $2810 \mathrm{msnm}$, las pruebas se realizaron en el Laboratorio del Centro de Transferencia Tecnológica para la Capacitación e Investigación en Control de Emisiones Vehiculares (CCICEV) en la ciudad de Quito-Ecuador.

\section{Caracterización del diésel/biodiésel.}

Para la caracterización se necesita de un laboratorio especializado. En la tabla 1 se encuentra los datos relevantes para la investigación comparados con los requerimientos en la Norma INEN 1489:2012 para diésel/biodiésel, los valores obtenidos cumplen a cabalidad con la norma, hay que notar que el contenido de azufre reducirá niveles de humo de los vehículos. También evidenciándose que el número de cetano regular 
y ayuda a obtener una mejor combustión. El aditivo se compone de un total $2882.52 \mathrm{mg} / \mathrm{kg}$ donde $28 \%$ es fósforo, $14 \%$ calcio, $12 \%$ zinc, $10 \%$ boro, $9 \%$ hierro, $8 \%$ manganeso, $6 \%$ aluminio, $5 \%$ vanadio, y el $8 \%$ restante incluye los 25 metales, estos fueron procesados mediante el Método de Inducción de Plasma Acoplada de Masas (ICPM). El aditivo se mezcló en la proporción de 12 mililitros por cada galón, de acuerdo a las recomendaciones del fabricante del producto.

Tabla 1: Propiedades de caracterización de las mezclas de diésel y biodiésel con aditivo

\begin{tabular}{|c|c|c|c|c|c|c|c|}
\hline $\begin{array}{l}\text { Propiedades del } \\
\text { combustible }\end{array}$ & Norma & $\begin{array}{l}\text { Diésel } \\
\text { Premium }\end{array}$ & $\begin{array}{l}\text { Diésel / } \\
10 \% \\
\text { biodiésel } \\
\text { (B10) }\end{array}$ & $\begin{array}{l}\text { Diésel / } \\
20 \% \\
\text { biodiésel } \\
\text { (B20) }\end{array}$ & $\begin{array}{c}\text { Diésel/ } \\
10 \% \\
\text { biodiésel - } \\
\text { aditivo } \\
\text { (B10A) }\end{array}$ & $\begin{array}{c}\text { Diésel / } \\
20 \% \\
\text { biodiésel - } \\
\text { aditivo } \\
\text { (B20A) }\end{array}$ & $\begin{array}{c}\text { Norma } \\
\text { INEN: } \\
\text { 1489:201 } \\
2\end{array}$ \\
\hline Número de cetano & $\begin{array}{c}\text { ASTM } \\
\text { D976 - } 06\end{array}$ & 51.7 & 53.2 & 53.2 & 51.9 & 52.5 & $45 \min$. \\
\hline Curva de destilación & \multirow[b]{2}{*}{ ASTM D88 } & \multirow[b]{2}{*}{336} & \multirow[b]{2}{*}{336} & \multirow[b]{2}{*}{343} & \multirow[b]{2}{*}{342} & \multirow[b]{2}{*}{343} & \multirow[b]{2}{*}{360 max. } \\
\hline $\begin{array}{l}\mathrm{T}_{90}-90 \% \text { evap. } \\
\left({ }^{\circ} \mathrm{C}\right)\end{array}$ & & & & & & & \\
\hline $\begin{array}{l}\text { Punto de } \\
\text { Inflamación }\left({ }^{\circ} \mathrm{C}\right)\end{array}$ & $\begin{array}{c}\text { ASTM D93 } \\
-16 a\end{array}$ & 61 & 63 & 66 & 61 & 64 & $51 \mathrm{~min}$. \\
\hline $\begin{array}{l}\text { Contenido de azufre } \\
\text { (ppm) }\end{array}$ & $\begin{array}{c}\text { ASTM } \\
\text { D4294 -16 } \\
\end{array}$ & 145.93 & 122.7 & 106.76 & 119.75 & 105.99 & 650 max. \\
\hline $\begin{array}{l}\text { Corrosión a la } \\
\text { lámina de cobre }\end{array}$ & $\begin{array}{c}\text { ASTM } \\
\text { D130-12 }\end{array}$ & $1 \mathrm{~A}$ & $1 \mathrm{~A}$ & $1 \mathrm{~A}$ & $1 \mathrm{~A}$ & $1 \mathrm{~A}$ & 3 \\
\hline $\begin{array}{l}\text { Viscosidad } \\
\text { cinemática a } 40 \stackrel{\circ}{ }= \\
\left(\mathrm{mm}^{2} / \mathrm{s}\right)\end{array}$ & $\begin{array}{c}\text { ASTM } \\
\text { D445 -15a }\end{array}$ & 3.528 & 3.445 & 3.459 & 3.459 & 3.283 & $2-5$ \\
\hline $\begin{array}{l}\text { Agua y sedimentos } \\
(\%)\end{array}$ & $\begin{array}{c}\text { ASTM } \\
\text { D1796 }-11\end{array}$ & $<0.05$ & $<0.05$ & $<0.05$ & $<0.05$ & $<0.05$ & $\begin{array}{l}0.05 \\
\text { máx. }\end{array}$ \\
\hline
\end{tabular}

\section{Comportamiento del motor diésel}

Se ha realizado una investigación de campo, de carácter explicativa, en la cual se busca determinar la diferencia en el nivel de opacidad y de la eficiencia mecánica, en la combustión de un motor de ciclo diésel, entre el diésel Premium comercializado en Ecuador. Para cada tipo de combustible, se han efectuado cinco mediciones, tomándose en consideración lo referenciado por Lozano-Ribas (2011), donde para un $95 \%$ de confianza y un 0.8 de diferencia mínima observable, manteniéndose un 0.8 de riesgo de cometer un error tipo II, se obtienen un mínimo de repeticiones de 4 . En la figura 1 está representado esquemáticamente el modelo del proceso de pruebas que se realizaron a los vehículos, las pruebas de par motor y potencia se realizaron en el dinamómetro de chasis marca Dynomite 3000-Lite de origen norteamericano; la opacidad se la trato en el opacímetro MAHA modelo MD02-LON de procedencia alemana. Los vehículos empleados en las pruebas fueron una camioneta MBT-50 $\left(2.5 \mathrm{~cm}^{3}\right)$ y otra marca GW Wingle $\left(2.8 \mathrm{~cm}^{3}\right)$, ambas con sistema de inyección diésel electrónica riel común (CRD-I). Las cuales poseen diferentes especificaciones técnicas, mostradas en la tabla 2.



Fig. 1: Flujograma del método experimental aplicado. 


\section{Protocolo de pruebas de opacidad}

La evaluación de la opacidad se realiza mediante ciclos de aceleración libre. La prueba consiste en que el vehículo se encuentre acondicionado y sin defectos, con el motor operando en ralentí y a temperatura normal de operación: oprimir completamente el acelerador en un tiempo menor a un segundo; mantener el acelerador completamente oprimido hasta que el motor alcance una velocidad superior a 3500, luego de alcanzarla, debe mantenerla de $2 \mathrm{~s}$ a 4 s hasta que el software de aplicación del equipo lo mida y precise, y después soltar el acelerador para que el motor regrese a su velocidad de ralentí; una vez suelto el acelerador deben transcurrir $15 \mathrm{~s}$ antes de iniciar el siguiente ciclo de aceleración libre. El vehículo debe recibir por lo menos cinco ciclos de ensayo empleando la secuencia anteriormente descrita. El primer ciclo permite que el operador de la prueba se familiarice con el movimiento adecuado del acelerador, y además retirar el hollín remanente que se pudiera acumular en el sistema de escape del vehículo durante su operación previa. Con los cuatro ciclos restantes se determinan los valores de humo máximos promediados en $0.5 \mathrm{~s}$, corregidos para cada uno de los cuatro ciclos de aceleración libre. Se realizaron cinco mediciones con cada tipo de combustible (según lo referido anteriormente). Los criterios de validación del ensayo del medidor de humo luego del ensayo no debe exceder $\pm 2 \%$ de opacidad para mediciones de humo, además la diferencia aritmética entre los valores mayores y menores de humo promedio máximo en 0.5 de los 5 ensayos no debe exceder de $5 \%$ de opacidad (INEN 2202, 2013; INEN 2207, 2002).

Tabla 2: Características principales de vehículos

\begin{tabular}{|l|l|l|}
\hline Modelo: & MBT-50 Doble Cabina Pick up & GW Wingle 5 Doble Cabina Pick up \\
\hline $\begin{array}{l}\text { Alimentación de } \\
\text { combustible: }\end{array}$ & $\begin{array}{l}\text { Inyección electrónica de diésel por riel } \\
\text { común CRDI }\end{array}$ & $\begin{array}{l}\text { Inyección electrónica de diésel por riel } \\
\text { común CRDI }\end{array}$ \\
\hline Tipo: & 16 válvulas, 4 cilindros en línea, DOHC & 16 válvulas, 4 cilindros en línea, DOHC \\
\hline Cilindrada: & $2499 \mathrm{~cm}^{3}$ & $2800 \mathrm{~cm}^{3}$ \\
\hline Potencia: & $117.3 \mathrm{~kW} @ 3500 \mathrm{rpm}$ & $80 \mathrm{~kW} @ 3600 \mathrm{rpm}$ \\
\hline Par motor: & $363 \mathrm{Nm}$ @ $1800 \mathrm{rpm}$ & $299.1 \mathrm{Nm}$ @ $1800-2000 \mathrm{rpm}$ \\
\hline Relación de compresión: & $18: 1$ & $17.7: 1$ \\
\hline
\end{tabular}

\section{Protocolo de pruebas de potencia y par motor}

La evaluación del rendimiento obtenido en el eje de salida a través del dinamómetro se realizó bajo la norma ISO 1585 e ISO 3173; siguiéndose el protocolo de pruebas: verificar que el diámetro de las ruedas sea igual o superior a rin 13 y que cumplan con la capacidad de peso establecida por el fabricante; colocar el vehículo de prueba en los rodillos del dinamómetro; verificar que el perfil del neumático esté libre de piedras en el labrado; descender el elevador y dejar apoyadas las ruedas sobre los rodillos; verificar la alineación del tren en donde se encuentra la transmisión con respecto a los rodillos del dinamómetro haciendo girar las ruedas a una velocidad máxima de 20 $\mathrm{km} / \mathrm{h}$; asegurar el vehículo con cintas para evitar que este se salga de los rodillos; revisar la seguridad del área de prueba; introducir en el software los datos técnicos referentes al vehículo a ser probado; comprobar la relación de cambio y de transmisión de la unidad, la cual debe ser en relación 1:1; asegúrese que la temperatura del motor es la normal de funcionamiento, caso contrario debe pasar un periodo de calentamiento del motor para alcanzar dicha temperatura; poner en funcionamiento el ventilador de refrigeración del banco de pruebas; iniciar la prueba de medición; acelerar el vehículo con el pedal a fondo en la marcha de prueba hasta alcanzar la velocidad deseada llamada "corte de rpm" (4500 - 6000 rpm); cuando se ha llegado al "corte de rpm", pisar el embrague, dejando la marcha puesta. El banco de potencia desacelera hasta detenerse (Corsini et al., 2016).

\section{Análisis de variables}

Tabla 3: Designación de nomenclatura para la formación de las combinaciones

\begin{tabular}{|c|c|c|c|}
\hline Factores & Niveles & Designación 1(tabla 4) & Designación 2 (tabla 5) \\
\hline \multirow{3}{*}{ Camioneta } & GW Wingle $\left(2.8 \mathrm{~cm}^{3}\right)$ & 1 & $\mathrm{~A} 2$ \\
\cline { 2 - 3 } & $\mathrm{MBT}-50\left(2.5 \mathrm{~cm}^{3}\right)$ & -1 & $\mathrm{~A}$ \\
\hline \multirow{4}{*}{ Combustibles } & Diésel & 1 & \multirow{2}{*}{-} \\
\cline { 2 - 3 } & $\mathrm{B} 10$ & 2 & \\
\cline { 2 - 4 } & $\mathrm{B} 20$ & 3 & \\
\cline { 2 - 4 } & $\mathrm{B} 10 \mathrm{~A}$ & 4 & \\
\cline { 2 - 4 } & $\mathrm{B} 20 \mathrm{~A}$ & 5 & \\
\hline
\end{tabular}

En el estudio se presenta el comportamiento de las variables dependientes: Potencia (kW), Par motor (Nm) y Opacidad (\%); al emplear distintas combinaciones de mezclas de combustibles en dos autos de referencia los cuales simulan el efecto de la variación de la cilindrada. Para el tratamiento estadístico de los resultados se toma en consideración las nomenclaturas que se representan en la tabla 3, además se emplea la propuesta 
de varios autores como la abordada por Valderrama (2005), y Vega et al., (2018); para determinar si entre los grupos experimentales existe diferencia significativa o no, se usó el análisis ANOVA, aplicándose pruebas de comparación múltiple de medias, para este caso la LSD (Least Significant Difference), para un $95 \%$ de confianza. Para el análisis y comparación de los resultados por medio de superficie de respuesta se forman tratamientos (combinaciones) que se muestran en la tabla 4 (Kolanjiappan, 2017; Guardia et al., 2018). Para evaluar si entre los tratamientos existen diferencias significativas se utiliza la designación presentada en la tabla 5.

Tabla 4: Tratamientos para el análisis de superficie de respuesta

\begin{tabular}{|c|c|c|}
\hline \multicolumn{3}{|c|}{ Formación de los tratamientos } \\
\hline No. & Autos & Combustibles \\
\hline 1 & -1 & 3 \\
\hline 2 & -1 & 1 \\
\hline 3 & 1 & 2 \\
\hline 4 & 1 & 5 \\
\hline 5 & -1 & 5 \\
\hline 6 & 1 & 1 \\
\hline 7 & 1 & 4 \\
\hline 8 & -1 & 2 \\
\hline 9 & 1 & 3 \\
\hline 10 & -1 & 4 \\
\hline
\end{tabular}

Tabla 5: Tratamientos para el análisis de diferencias significativas

\begin{tabular}{|c|c|c|}
\hline \multicolumn{3}{|c|}{ Formación de los tratamientos } \\
\hline No. & Autos & Combustibles \\
\hline 1 & A1 & Diésel \\
\hline 2 & A1 & B10 \\
\hline 3 & A1 & B20 \\
\hline 4 & A1 & B10A \\
\hline 5 & A1 & B20A \\
\hline 6 & A2 & Diésel \\
\hline 7 & A2 & B10 \\
\hline 8 & A2 & B20 \\
\hline 9 & A2 & B10A \\
\hline 10 & A2 & B20A \\
\hline
\end{tabular}

\section{RESULTADOS Y DISCUSIÓN}

A partir de los protocolos de prueba especificada en la metodología, se obtienen los resultados de potencia, par motor y opacidad, permitiéndose la evaluación de los combustibles experimentados.

\section{La potencia de los motores}

En la figura 2 se representa la potencia para los motores de las camionetas con mezclas B10, B20, B10A, B20A y diésel a diferentes revoluciones. La tendencia de la potencia para las camionetas es mayor con el uso del diésel obteniéndose su máximo valor a $4300 \mathrm{rpm}$ para MBT-50 y a $2500 \mathrm{rpm}$ para GW Wingle. En la tabla 6 y figura 3, se representa la prueba de múltiple rango, y gráfico de caja y bigotes para la variable dependiente (potencia), en la misma se aplica el procedimiento de diferencia mínima significativa (LSD) de Fisher con un nivel del $95.0 \%$ de confianza. Se evidencia que existe diferencia significativa entre los autos, siendo el MBT50 el de mejor resultado; además se aprecia una misma tendencia en ambos vehículos a mejorar la potencia máxima con el empleo de los combustibles diésel y biodiésel más $10 \%$ aditivo (B10A).

a)

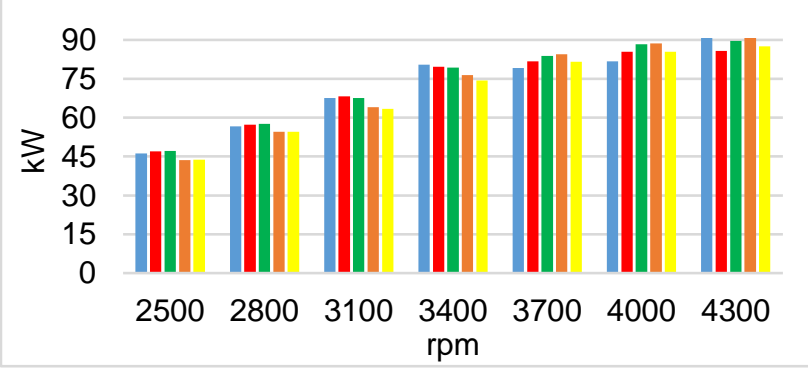

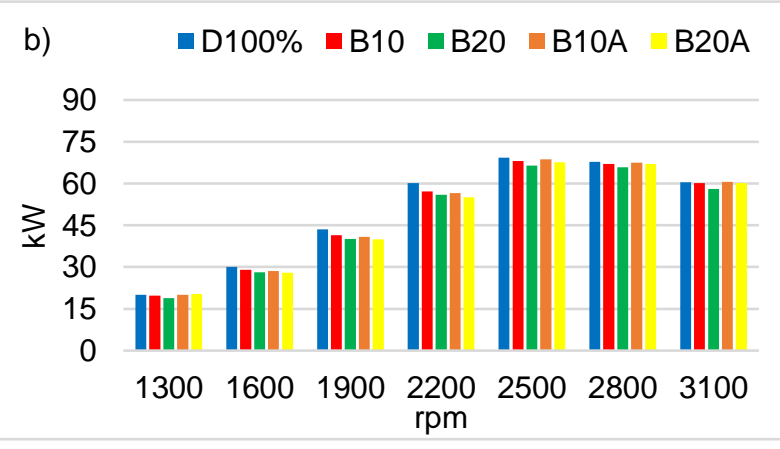

Fig. 2: Potencia promedio a diferentes rpm con diésel y mezclas, camionetas MBT-50 a) y GW Wingle b)

Por otra parte, la potencia de GW Wingle del combustible B20 obtuvo la mayor reducción en $2.7 \%$ con relación al diésel seguido del B10, resultado que concuerda con los datos obtenidos por Corsini et al., (2016) donde a medida que se incrementa el biodiesel disminuye la potencia. En el caso de la MBT-50 se obtuvo la mayor reducción de potencia de $5.4 \%$ al aplicar la mezcla B10 respecto al combustibles diésel. Los resultados de Afzal et al., (2018) muestran que los aditivos sintéticos (BHT, TBHQ y PG) fueron más eficientes que los extractos antioxidantes naturales (meta-extractos nólicos), cuestión esta que corrobora los resultados obtenidos, donde las mezclas tratadas con aditivos poseen mejor prestación en ambos vehículos. La figura 4 representa la relación que posee la potencia en función del tipo de combustible y los autos, definiéndose el modelo matemático que los relaciona, los mejores valores (máximo) se obtienen al disminuir el porcentaje de biodiesel, llegándose al valor óptimo de $89,26 \mathrm{~kW}$. En la figura 5, se aprecia como el factor autos es altamente significativo. 
Tabla 6: Tratamientos para el análisis de diferencias significativas.

\begin{tabular}{|l|l|l|c|}
\hline & Casos & \multicolumn{1}{|c|}{ Media } & $\begin{array}{c}\text { Grupos } \\
\text { Homogéneos }\end{array}$ \\
\hline A2B20 & 3 & 67.94 & $\mathrm{X}$ \\
\hline A2B10 & 3 & 68.92 & $\mathrm{X}$ \\
\hline A2B20A & 3 & 68.94 & $\mathrm{X}$ \\
\hline A2B10A & 3 & 69.4867 & $\mathrm{XX}$ \\
\hline A2Diésel & 3 & 69.8033 & $\mathrm{X}$ \\
\hline A1B10 & 3 & 85.61 & $\mathrm{X}$ \\
\hline A1B20A & 3 & 87.0 & $\mathrm{X}$ \\
\hline A1B20 & 3 & 89.5267 & $\mathrm{X}$ \\
\hline A1B10A & 3 & 90.5333 & $\mathrm{X}$ \\
\hline A1Diésel & 3 & 90.56 & $\mathrm{X}$ \\
\hline
\end{tabular}

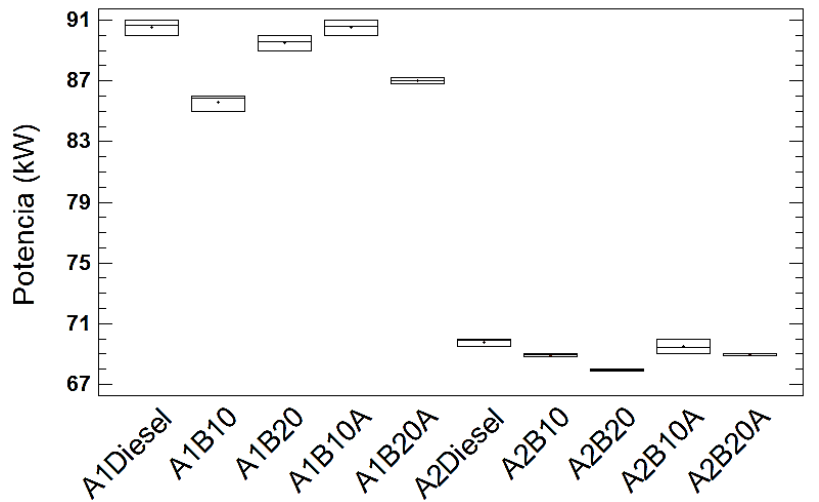

Fig. 3: Gráfico comparativo entre los vehículos con distintos combustibles



Potencia $=79.49-10.02 * \mathrm{~A}-0.2 * \mathrm{~B}+0.06 * \mathrm{~A} * \mathrm{~B}$

Fig. 4: Superficie de respuesta para el análisis de la potencia de las camionetas



Fig. 5: Gráfico de significación de los parámetros para la potencia

\section{El par motor}

En la figura 6 se presenta el par motor para los motores con mezclas B10, B20, B10A, B20A y diésel a diferentes revoluciones. La tendencia para ambas camionetas es mayor con el uso del diésel obteniéndose su máximo valor a $3400 \mathrm{rpm}$ para MBT-50 y a $2500 \mathrm{rpm}$ para GW Wingle. El par motor en la figura 6, muestra para la MBT-50 en promedio un decrecimiento del 0.94 y $1.36 \%$ utilizando mezclas de B10 y B20 respectivamente, en comparación a lo obtenido al utilizar diésel. El mayor par en la camioneta GW Wingle lo obtuvo el diésel a $2500 \mathrm{rpm}$ con $268.99 \mathrm{Nm}$, los combustibles en mezcla a la misma revolución obtuvieron una disminución de torque de 2.37 y $4.50 \%$ con mezcla B10 y B20 respectivamente. Kousoulidou et al., (2010) concluyó que la mezcla de biodiesel en diésel derivado del petróleo a $10 \%$ en volumen cambia las propiedades del combustible en un grado que puede afectar las características de combustión, como el inicio del encendido, además, Campaña et al., (2016) en sus resultados obtuvo que el diésel presentó un mayor par motor en comparación con el B10, lo que coincide con los resultados de la presente experimentación (Zare et al., 2016).

En la tabla 7 y figura 7 , se representa la prueba de múltiple rango y gráfico de caja y bigotes para la variable dependiente par motor, en la misma se aplica el procedimiento de diferencia mínima significativa (LSD) de Fisher con un nivel del $95.0 \%$ de confianza. Se concluye que existe diferencia significativa entre los autos, siendo el GW Wingle con mejores resultados; además se aprecia una misma tendencia en ambos vehículos a mejorar el par motor con el empleo de los combustibles diésel y Biodiésel (B10A). La figura 8 representa la relación que posee el par motor en función del tipo de combustible y los autos, definiéndose el modelo matemático que los relaciona, donde los mejores valores (máximo) se obtienen al disminuir el porcentaje de biodiesel, llegándose al valor óptimo de 267,68 Nm; destacándose la influencia del combustible y los autos de manera significativa como se muestra en la figura 9 del Pareto. 

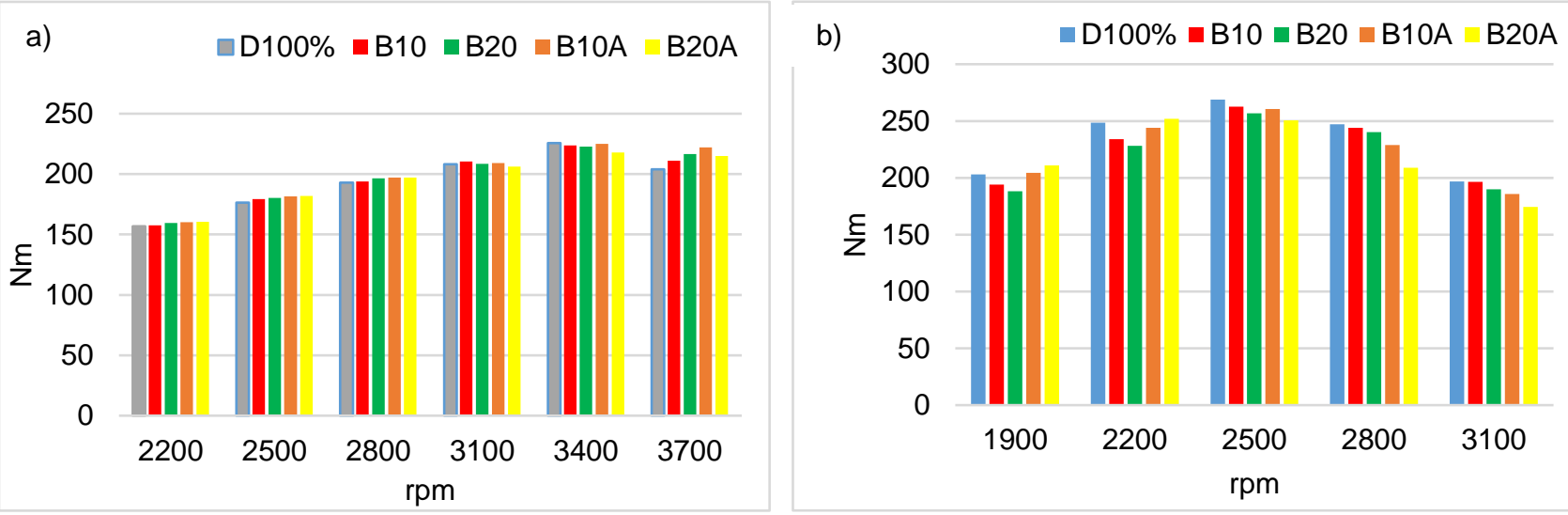

Fig. 6: Par motor promedio a diferentes rpm con diésel y mezclas, camionetas MBT-50 a) y GW Wingle b)

Tabla 7: Tratamientos para el análisis de diferencias significativas. Método: 95,0 porcentaje LSD

\begin{tabular}{|l|l|l|c|}
\hline & Casos & Media & Grupos Homogéneos \\
\hline TA1B20A & 3 & 217.7 & $\mathrm{X}$ \\
\hline TA1B20 & 3 & 222.543 & $\mathrm{X}$ \\
\hline TA1B10 & 3 & 223.523 & $\mathrm{X}$ \\
\hline TA1B10A & 3 & 224.9 & $\mathrm{X}$ \\
\hline TA1Diesel & 3 & 225.567 & $\mathrm{X}$ \\
\hline TA2B20 & 3 & 256.86 & $\mathrm{X}$ \\
\hline TA2B20A & 3 & 259.0 & $\mathrm{X}$ \\
\hline TA2B10A & 3 & 262.467 & $\mathrm{X}$ \\
\hline TA2B10 & 3 & 262.54 & $\mathrm{X}$ \\
\hline TA2Diesel & 3 & 268.93 & $\mathrm{X}$ \\
\hline
\end{tabular}

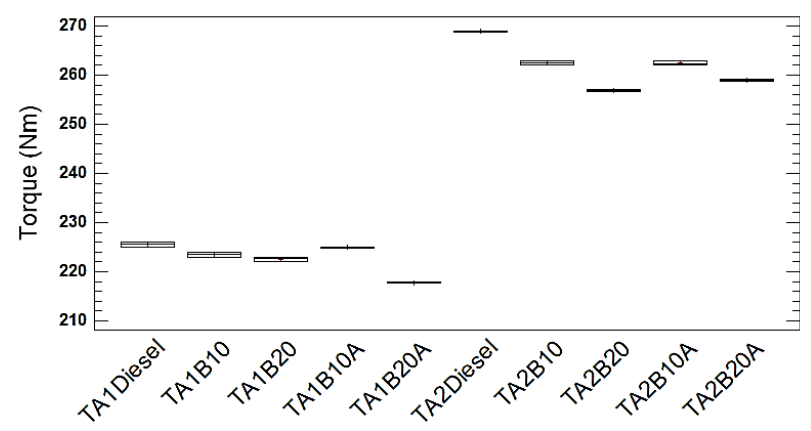

Fig. 7: Gráfico comparativo del par motor entre los vehículos con distintos combustibles

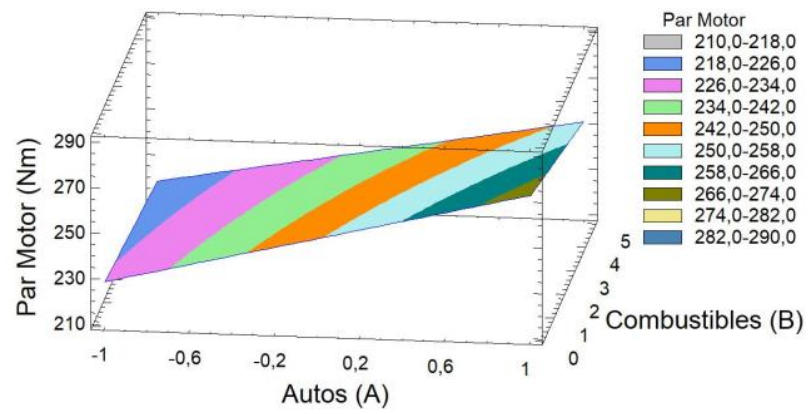

Par Motor $=249.42+22.12 * \mathrm{~A}-2.66 * \mathrm{~B}-1.2 * \mathrm{~A} * \mathrm{~B}$

Fig. 8: Superficie de respuesta para el análisis del par motor de las camionetas

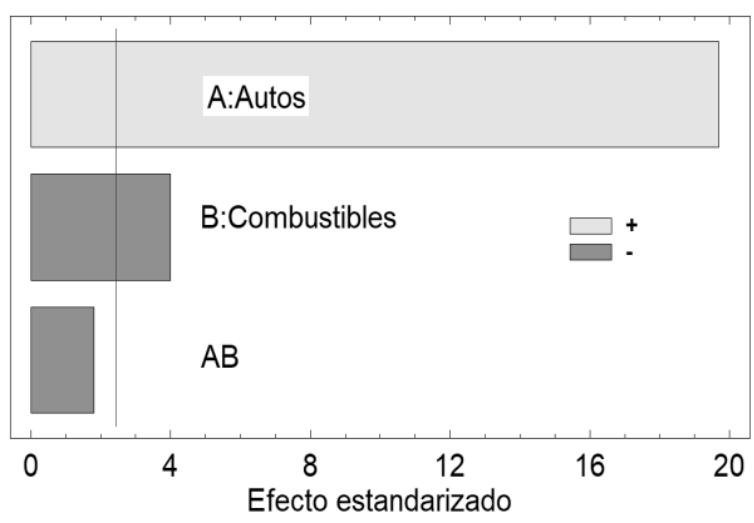

Fig. 9: Gráfico de significación de los parámetros del par motor

\section{Resultados de Opacidad.}

En la figura 10 se presenta la gráfica de columnas con el promedio de cada uno de los combustibles empleados en la camioneta MBT-50, donde se evidencio que con el combustible B10 se logra la mayor reducción de la opacidad con un $20 \%$ y el B20 con un $8.8 \%$ el que reduce menos con respecto al diésel. En el caso de la camioneta GW Wingle se presenta la mayor reducción de opacidad con el combustible B20 en un $38 \%$ y el B10 con $20.34 \%$ el que reduce menos con respecto al diésel. En la tabla 8 y figura 11, se representa la prueba de múltiple rango y gráfico de caja y bigotes para la variable dependiente Opacidad, en la misma se aplica el procedimiento de diferencia mínima significativa (LSD) de Fisher con un nivel del 95.0\% de confianza. Se muestra que existe diferencia significativa entre los autos, siendo el GW Wingle el de mejor resultado; además se aprecia una misma tendencia en ambos vehículos a mejorar la opacidad con el empleo de biodiesel, resultado que coincide con los de Yépez et al., (2018) donde obtuvo que los valores de opacidad con el uso de biocombustible son menores al comparar con el diésel. 


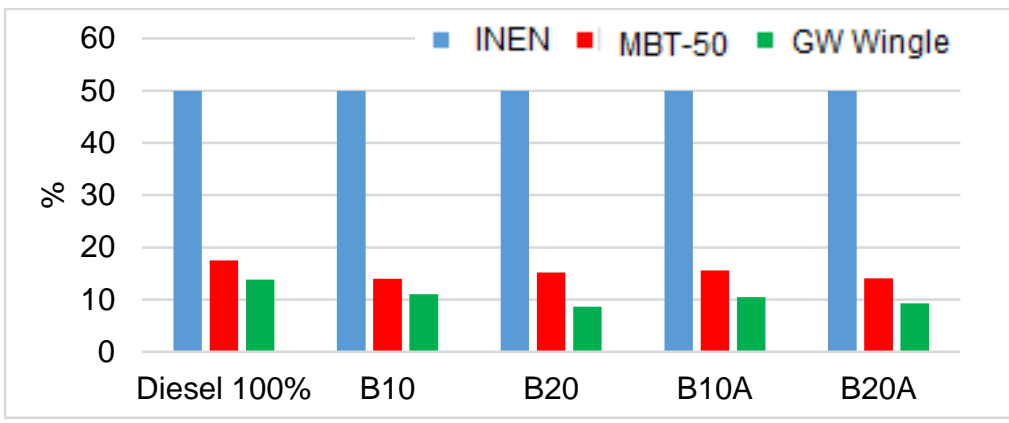

Fig. 10: Opacidad promedio con diésel y mezclas, camionetas MBT-50 y GW Wingle

Tabla 8: Tratamientos para el análisis de diferencias significativas. Método: 95,0 porcentaje LSD

\begin{tabular}{|l|l|l|l|}
\hline & Casos & Media & Grupos Homogéneos \\
\hline OA2B20 & 3 & 8.55667 & $\mathrm{X}$ \\
\hline OA2B20A & 3 & 9.3 & $\mathrm{X}$ \\
\hline OA2B10A & 3 & 10.5 & $\mathrm{X}$ \\
\hline OA2B10 & 3 & 11.0 & $\mathrm{X}$ \\
\hline OA2Diesel & 3 & 13.81 & $\mathrm{X}$ \\
\hline OA1B10 & 3 & 14.0 & $\mathrm{X}$ \\
\hline OA1B20A & 3 & 14.0333 & $\mathrm{X}$ \\
\hline OA1B10A & 3 & 15.5333 & $\mathrm{X}$ \\
\hline OA1B20 & 3 & 15.9567 & $\mathrm{X}$ \\
\hline OA1Diesel & 3 & 17.5 & $\mathrm{X}$ \\
\hline
\end{tabular}

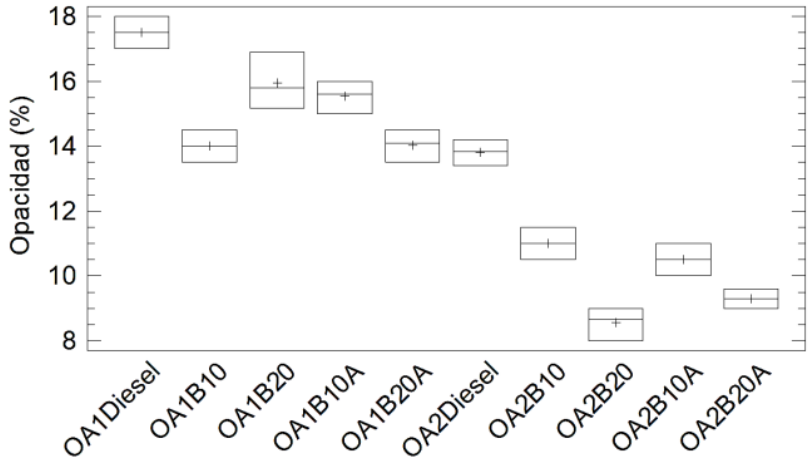

Fig. 11: Gráfico comparativo entre las camionetas con distintos combustibles

Los resultados obtenidos por Kolanjiappan (2017) con combustible B100 y B20 aditivados con antioxidantes revelaron que la opacidad es casi persistente e imperceptible en un motor de potencia de freno de $5.9 \mathrm{~kW}$ y se incrementa a altas cargas para todas las mezclas, estos resultados concuerdan con los obtenidos en el presente estudio. La figura 12 representa la relación que posee la opacidad en función del tipo de combustible y los autos, definiéndose el modelo matemático que los relaciona, donde los mejores valores (mínimos) se obtienen con el aumento del porcentaje de biodiesel, llegándose al valor óptimo de 8,7 \%; destacándose la influencia de los autos seguido del tipo de combustible como se muestra en la figura 13 del Pareto.



Opacidad $=15.18-1.65 * \mathrm{~A}-0.74 * \mathrm{~B}-0.22 * \mathrm{~A} * \mathrm{~B}$

Fig. 12: Superficie de respuesta para el análisis de la opacidad

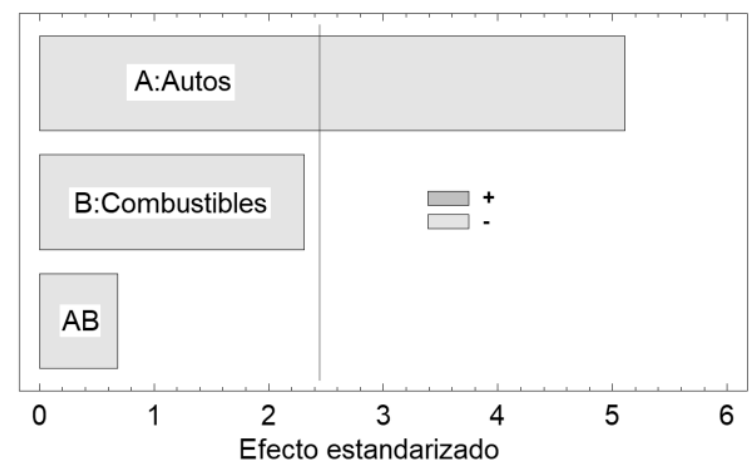

Fig. 13: Gráfico de significación de los parámetros de opacidad

\section{CONCLUSIONES}

De acuerdo a los resultados de este estudio, y su análisis se pueden extraer los siguientes datos relevantes: 1) La mezcla de combustible B10 es la más óptima logrando mantener tanto el par motor como la potencia sin disminuciones significativas para un $95 \%$ de confianza, $2.37 \%$ en el par motor y una reducción de 1.37 $\%$ en potencia con respecto al diésel estos datos fueron obtenidos en la GW Wingle; en cambio la MBT-50 se obtuvo una reducción de $0.94 \%$ en el par motor con el combustible B10 y una reducción del $1.21 \%$ con la mezcla de combustible B20, esto concluye que a medida que se aumenta la concentración de biodiésel en la mezcla la eficiencia térmica disminuye, esto debido aumento de viscosidad y densidad del combustible. 2) Se apreció con las pruebas realizadas de opacidad, que la mezcla de combustible B20 con respecto al diésel 
fósil redujo la opacidad en un $37.35 \%$ en la GW Wingle, en cambio en la MBT-50 se obtuvo una reducción del $4 \%$ con la mezcla de combustible B10; deduciéndose que los combustibles con mezclas de biodiésel sí reducen la emanación de hollín u opacidad de los vehículos, contribuyéndose a minimizar la contaminación. En general la opacidad disminuye a medida que se incrementa el biodiésel en la mezcla, debido a que la emisión de material particulado disminuye.

\section{REFERENCIAS}

Afzal, S., M.W. Mumtaz y otros seis autores, Exhaust Emission Profiling of Fatty Acid Methyl Esters and Nox Control Studies Using Selective Synthetic and Natural Additives, doi: 10.1007/s10098-018-1489-3, Clean Tech. and Envir. Policy, 20(3), 589-601 (2018)

Basha, S.A. y K.R. Gopal, A Review of the Effects of Catalyst and Additive on Biodiesel Production, Performance, Combustion and Emission Characteristics, doi: 10.1016/j.rser.2011.08.036, Renew. and Sust. Energy Rev., 16(1), 711. 717 (2012)

Campaña, G.G.R., J.A.C. Reyes y A. X. E. Quezada, Assessment of Torque and Power In an Internal Combustion Engine Using Partial Mixtures of Biodiesel, J. Eng. and Ed., 12(20), 23-31 (2016)

Çelik, M., H. Solmaz y H.S. Yücesu, Examination of the Effects of Organic Based Manganese Fuel Additive on Combustion and Engine Performance, doi:10.1016/j.fuproc.2015.08.002, Fuel Proc. Tech., 139, 100-107 (2015)

Chen, Y., J. Ma y otros cinco autores, Emissions of automobiles fueled with alternative fuels based on engine technology: a review, doi: 10.1016/j.jtte.2018.05.001, J. Traffic and Transportation Engineering, 5(4), 318-334 (2018)

Corsini, A., R. Di Antonio y otros cuatro autores, Performance analysis of a common-rail Diesel engine fuelled with different blends of waste cooking oil and gasoil, Energy Procedia, 101, 606-613 (2016)

Guardia, Y., J. Márquez y otros cuatro autores, Mejoras a la asignatura Diseño Estadístico de Experimentos para estudiantes de la carrera de Ingeniería Mecánica, Revista ESPACIOS, 39(30), 10-26 (2018)

INEN 2202: Gestión Ambiental. Aire. Vehículos Automotores. Determinación de la Opacidad de Emisiones de Escape de Motores de Diésel Mediante la Prueba Estática. Método de Aceleración Libre, Primera, Quito-Ecuador (2013)

INEN 2207: Gestión Ambiental. Aire. Vehículos Automotores. Límites Permitidos de Emisiones por Fuentes Móviles Terrestres de Diésel, Primera, Quito-Ecuador (2002)

Kolanjiappan, V., Reduction of Amine and Biological Antioxidants on Nox Emissions Powered by Mango Seed Biodiesel, doi: 10.17533/udea.redin.n84a06, Rev. Fac. Ing. Univ. Antioquia, (84), 46-54 (2017)

Kousoulidou, M., G. Fontaras, L. Ntziachristos y Z. Samaras, Biodiesel blend effects on common-rail diesel combustion and emissions, doi: 10.1016/j.fuel.2010.06.034, Fuel, 89(11), 3442-3449 (2010)

Lenin, M.A., M.R. Swaminathan y G. Kumaresan, Performance and Emission Characteristics of A DI Diesel Engine with a Nanofuel Additive, doi: 10.1016/j.fuel.2013.03.042, Fuel, 109, 362-365 (2013)

Lozano-Rivas, W.A., Determinación del número mínimo de observaciones en investigación, obviando las estimaciones de la varianza de datos, Revista de Didáctica Ambiental, 10, 54-61 (2011)

Mena, L., M. Román y otros cuatro autores, Estudio de Rugosidad por Análisis de Fourier de las Toberas de Inyectores en Sistemas Riel Común (CRDI), Ingeniare, 26(4), 654-662 (2018)

Mofijur, M, H.H. Masjuki y otros cuatro autores, Comparative Evaluation of Performance and Emission Characteristics of Moringa Oleifera and Palm Oil Based Biodiesel In a Diesel Engine, doi: 10.1016/j.indcrop.2013.12.011, Ind. Crop. Prod., 53, 78-84 (2014)

Moghaddam, M.S. y A. Z. Moghaddam, Performance and Exhaust Emission Characteristics of A Cl Engine Fueled with Diesel-Nitrogenated Additives, doi: 10.1016/j.cherd.2014.01.009, Chem. Eng. Research and Design, 92(4), 720-726 (2014)

Mumtaz, M.W., A. Adnan y otros cinco autores, Response Surface Methodology: an Emphatic Tool for Optimized Biodiesel Production Using Rice Bran and Sunflower Oils, doi:10.3390/en5093307, Energies, 5, 3307-3328 (2012)

Ozsezen, A.N., M. Canakci, A. Turkcan y C. Sayin, Performance and Combustion Characteristics of A DI Diesel Engine Fueled with Waste Palm Oil and Canola Oil Methyl Esters, doi: 10.1016/j.fuel.2008.09.023, Fuel, 88, 629-636 (2009)

Payri, R., F.J. Salvador, J. Gimeno y G. Bracho, The Effect of Temperature and Pressure on Thermodynamic Properties of Diesel and Biodiesel Fuels, doi: 10.1016/j.fuel.2010.11.015, Fuel, 90(3), 1172-1180 (2011)

Qi, D.H., L. M. Geng, H. Chen, Y.Z.H. Bian y J. Liu, Combustion and Performance Evaluation of A Diesel Engine Fueled with Biodiesel Produced from Soybean Crude Oil, Renew Energy, 34, 2706-2713 (2009)

Rocha-Hoyos, J., L. E. Tipanluisa, V. D. Zambrano y A. A. Portilla, Estudio de un Motor a Gasolina en Condiciones de Altura con Mezclas de Aditivo Orgánico en el Combustible, Inf. Tecnol., 29(5), 325-334 (2018)

Rocha-Hoyos, J., L. E. Tipanluisa, S. W. Reina y C. R. Ayabaca, Evaluación del Sistema de Tracción en un Vehículo Eléctrico Biplaza de Estructura Tubular, doi: 10.4067/S0718-07642017000200004, Inf. Tecnol., 28(2), 29-36 (2017) 
Thiyagarajan, S., V. Geo, L.J. Martin y B. Nagalingam, Simultaneous Reduction of NO-Smoke-CO Emission in A Biodiesel Engine using Low-Carbon Biofuel and Exhaust After-Treatment System, doi: 10.1007/s10098-016-1326-5, Clean Techn. Environ. Policy, 19, 1271-1283 (2017)

Thoo, W.J., A. Kevric y otros cuatro autores, Characterization of Ignition Delay Period for a Compression Ignition Engine Operating on Blended Mixtures of Diesel and Gasoline, doi: 10.1016/j.applthermaleng.2014.01.066, Applied Thermal Eng., 66(1-2), 55-64 (2014)

Valderrama, J.O., Principales Aspectos sobre la Preparación de un Artículo para ser Publicado en una Revista Internacional de Corriente Principal, doi: 10.4067/S0718-07642005000200002, Inf. Tecnol., 16(2), 3-14 (2005)

Varatharajan, K., M. Cheralathan y R. Velraj, Mitigation Of Nox Emissions from a Jatropha Biodiesel Fuelled DI Diesel Engine using Antioxidant Additives, Fuel, 90, 2721-2725 (2011)

Vega, W.H., E.A. Llanes-Cedeño, J.V. Molina y J.C. Rocha-Hoyos, Revisión de las Características de Modelado y Optimización para el Diseño del Sistema de Suspensión Macpherson, Inf. Tecnol., 29(6), 221-233 (2018)

Wei, L., C.S. Cheung y Z. Ning, Effects of Biodiesel-Ethanol and Biodiesel-Butanol Blends on the Combustion, Performance and Emissions of a Diesel Engine, doi: 10.1016/j.energy.2018.05.049, Energy, 155, 957-970 (2018)

Yépez, C.N.M., R.I. Navarrete y otros tres autores, Cuantificación de la Opacidad en Motores Electrónicos Diésel usando Diésel y Biodiésel. Ingenius, 19, 102-107 (2018)

Zare, A., M.N. Nabi y otros cinco autores, The Effect of Triacetin as a Fuel Additive to Waste Cooking Biodiesel on Engine Performance and Exhaust Emissions, doi: 10.1016/j.fuel.2016.06.039, Fuel, 182, 640-649 (2016) 